\title{
New Reconstructions on Silicon (111) Surfaces
}

\section{Citation}

Becker, R. S., J. A. Golovchenko, G. S. Higashi, and B. S. Swartzentruber. 1986. New

Reconstructions on Silicon (111) Surfaces. Physical Review Letters 57, no. 8: 1020-1027. doi:10.1103/physrevlett.57.1020.

\section{Published Version}

doi:10.1103/PhysRevLett.57.1020

\section{Permanent link}

http://nrs.harvard.edu/urn-3:HUL.InstRepos:29407041

\section{Terms of Use}

This article was downloaded from Harvard University's DASH repository, and is made available under the terms and conditions applicable to Other Posted Material, as set forth at http:// nrs.harvard.edu/urn-3:HUL.InstRepos:dash.current.terms-of-use\#LAA

\section{Share Your Story}

The Harvard community has made this article openly available.

Please share how this access benefits you. Submit a story.

Accessibility 


\title{
New Reconstructions on Silicon (111) Surfaces
}

\author{
R. S. Becker, J. A. Golovchenko, G. S. Higashi, and B. S. Swartzentruber \\ AT\&T Bell Laboratories, Murray Hill, New Jersey 07974 \\ (Received 4 June 1986)
}

\begin{abstract}
We report the first observation of a class of new reconstructions on clean silicon (111) surfaces. The surfaces are prepared by a combination of laser and thermal annealing and the new structures are studied with a tunneling microscope. $c-(4 \times 2)$ and $(2 \times 2)$ regions populate the ordered parts of the surface directly after laser annealing. Subsequent partial thermal anneals result in a surface containing $(5 \times 5),(7 \times 7),(9 \times 9)$, and other intermediate structures. These observations' bearing on the connection between germanium and silicon reconstructions is discussed.
\end{abstract}

PACS numbers: $61.16 . \mathrm{Di}, 68.35 . \mathrm{Bs}$

Six years ago reports appeared in the literature showing that exposure of clean reconstructed $\mathrm{Si}(111)$ $(7 \times 7)$ surfaces to $Q$-switched laser pulses results in a transformation such that $(1 \times 1)$ patterns are observed with low-energy electron diffraction. ${ }^{1,2}$ It was suggested that the high cooling rate associated with laser annealing may have resulted in the previously observed high-temperature $(1 \times 1)$ phase remaining metastably at the surface. A number of subsequent studies have led their authors to draw somewhat different conclusions. Channeling, ${ }^{3}$ photoemission, ${ }^{4}$ low-energy electron-diffraction (LEED) ${ }^{5}$ and infrared-absorption studies of hydrogen chemisorption ${ }^{6}$ have been interpreted in terms of a "disordered $(7 \times 7)$ " surface structure, or relaxed bulk structure, with perhaps some $(2 \times 1)$ reconstruction ${ }^{7}$ similar to that observed on cleaved crystals.

We have restudied this problem using the tunneling microscope which has recently contributed significant new information about semiconductor surface reconstructions. $^{8}$ Our results indicate that completely new and unanticipated structures are present on the laserannealed silicon surface. Further new structures can be induced with additional surface processing. Each of these new structures bears a special relationship to already known surface phases thus shedding light on the entire family of semiconductor reconstructions.

Sample preparation and characterization and the ultrahigh-vacuum tunneling microscope used in this study have been previously described. ${ }^{9} \mathrm{~A}(7 \times 7)$ surface was obtained and verified by both LEED and tunneling images. The sample was then exposed in the ultrahigh-vacuum chamber to an optical pulse from a $Q$-switched, frequency-doubled, Nd:YAlG (yttrium aluminum garnet) laser (530 nm, 15-nsec pulse) operated in a pseudo-Gaussian transverse mode. A spot on the sample several millimeters in diameter was irradiated at a fluence of $\sim 1 \mathrm{~J} / \mathrm{cm}^{2}$. This is the kind of dose required for melting several thousand angstroms of silicon back from the surface and characterizes the laser-annealing regime. ${ }^{10,11}$ The rapid melting is followed by rapid recrystallization where the bulk crystal serves as the template for the ensuing liquid- phase epitaxial regrowth. Regrowth velocities can exceed $1 \mathrm{~m} / \mathrm{sec}$.

A LEED measurement showed that the above treatment transformed the surface to an apparent $(1 \times 1)$ structure. Careful observation indicated some slight diffuse intensity enhancement at half-order positions. ${ }^{7}$ A series of tunneling images of the surface were then taken over the transformed region of the surface. The resulting surfaces had atomically flat regions, some extending thousands of angstroms laterally. Atomic steps were detected but they were very disordered with respect to orientation. Reconstructed steps like those observed on equilibrium surfaces ${ }^{9}$ were never observed. Step heights were measured to be $3.1 \AA$ in agreement with the double-layer step height on the equilibrium surface. Figure 1 shows a high-resolution tunneling image of a $100 \times 100-\AA^{2}$ region of the surface that not only illustrates the rather helter-skelter structure of the steps but also shows several small domains of $c-(4 \times 2)$ and $(2 \times 2)$ reconstruction. Such regions rarely consist of more than several unit cells which are of course distributed over the various twin configurations consistent with the threefold symmetry of the underlying surface. It is probably the extremely small size of these structures that has not allowed their previous identification by diffraction methods. We have also never observed these structures on non-laserquenched silicon (111) surfaces.

Previous studies ${ }^{2}$ have shown that subsequent slow thermal anneal to approximately $750^{\circ} \mathrm{C}$ induces an apparent complete recovery of the $(7 \times 7)$ LEED spots. Before achieving this condition we decided to study the tunneling images for a surface that was heated to only $600^{\circ} \mathrm{C}$ for a minute. Here the three and four sevenths spots were just beginning to intensify but a great deal of apparent diffuse scattering and streakiness remained in the diffraction pattern. After waiting several hours for the thermal drifts to stabilize in the tunneling microscope we obtained a series of tunneling images that showed remarkable and unexpected new features at the surface.

Figure 2 shows one such tunneling image which contains several features reminiscent of equilibrium 


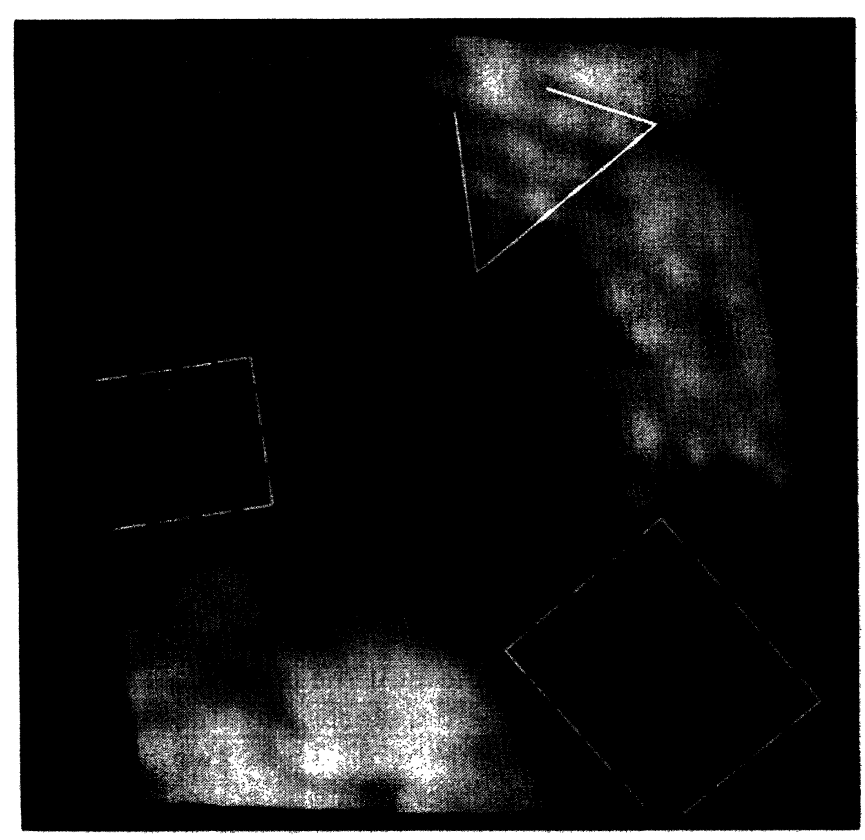

FIG. 1. Tunneling image of a laser-annealed Si (111) surface. Dashed lines enclose regions of $c-(4 \times 2)$ reconstruction and solid lines delineate $(2 \times 2)$ regions. Shading is by height with light areas high and dark areas low. The two light-gray regions in the lower left and upper right are double-layer atomic steps $(3.1 \AA)$ up from the gray regions containing the $c-(4 \times 2)$. The area shown is $-95 \times 105 \AA^{2}$. The tip bias was $-0.5 \mathrm{~V}$ and tunnel current $1.0 \mathrm{nA}$. Image acquisition time is $2 \mathrm{~min}$.

$(7 \times 7)$ surfaces. In fact complete and partial unit cells of this type are seen distributed across the $100 \times 100$ $\AA^{2}$ imaged area. One such region is indicated by dashed lines to help the reader. Much more interestingly there appears for the first time a complete $(9 \times 9)$ unit cell, which is indicated by a solid-line border. This structure is very similar to the $(7 \times 7)$ in that it is rhombic and contains an array of ordered protrusions symmetrically distributed across the cell's short diagonal. There are, however, twenty of these protrusions in the $(9 \times 9)$ as compared with twelve in the $(7 \times 7)$. Three of the four corners of the $(9 \times 9)$ cell are bounded by deep corner holes characteristic of the $(7 \times 7)$ structure. A region of the surface where the protrusions array themselves in a $(\sqrt{3} \times \sqrt{3})-R 30$ pattern is also indicated in the upper-left-hand corner.

The final tunneling image we present here is shown in Fig. 3. It shows another region on the same surface which in addition to $(7 \times 7)$ pieces contains $(5 \times 5)$ regions. Such complete unit cells would contain six protrusions, three in each triangular half of the cell. Again deep corner holes are the natural corner boundaries. Much of the top riser of the atomic step visible in the lower part of the figure is composed of $(5 \times 5)$ unit-cell halves.

The annealing experiment was completed by heating

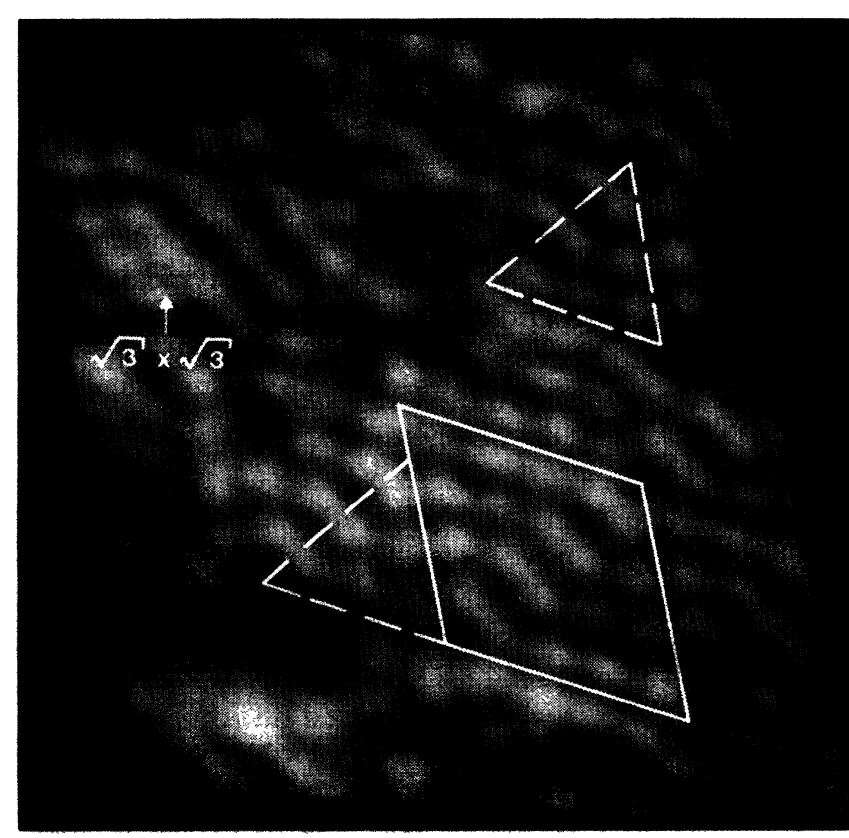

FIG. 2. Tunneling image of a laser-annealed Si (111) surface subsequently thermally annealed at $600^{\circ} \mathrm{C}$. The dashed lines indicate partial $(7 \times 7)$ cells. The solid line surrounds a $(9 \times 9)$ cell. A $(\sqrt{3} \times \sqrt{3})-R 30$ region is indicated in the upper left. The area depicted is $-95 \times 105 \AA^{2}$ and the gray areas are assigned as in Fig. 1.

of the sample for several minutes at $800^{\circ} \mathrm{C}$. The complete $(7 \times 7)$ LEED pattern was recovered with very sharp diffraction spots and an extremely low diffuse

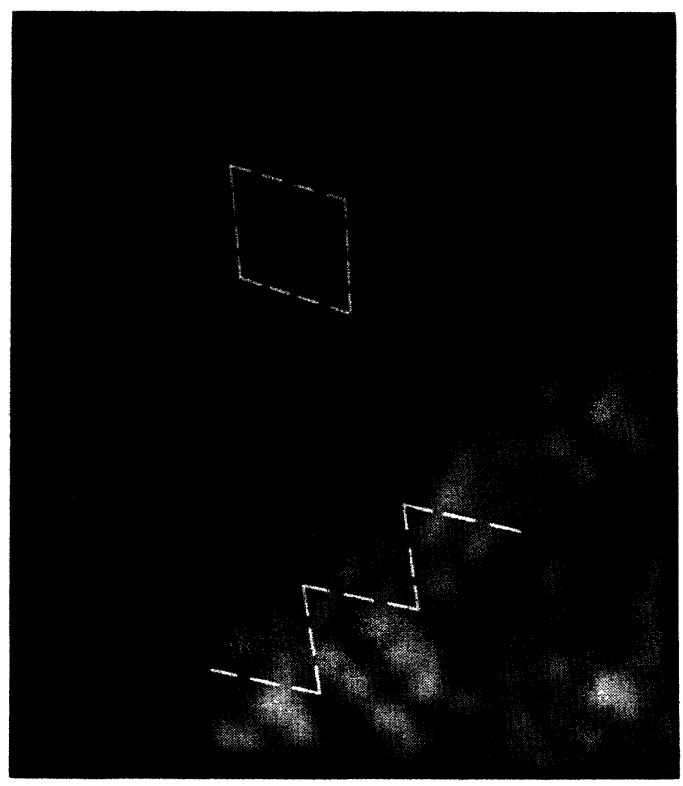

FIG. 3. Tunneling image of a laser-annealed Si (111) surface subsequently thermally annealed at $600^{\circ} \mathrm{C}$. The area depicted is $-95 \times 105 \AA^{2}$. The dashed lines depict $(5 \times 5)$ cells and half-cells which are prominent along the step edge at the lower right. Gray levels assigned as in Fig. 1. 
background. The tunneling images for this surface showed a high-quality pattern with continuous $(7 \times 7)$ regions extending several thousand angstroms before atomic steps or significantly disordered regions were encountered. The laser-annealing pretreatment thus results in a $(7 \times 7)$ surface that is flat and periodic over distances ten times larger than we have typically observed on sputtered and thermally annealed surfaces.

We begin our discussion of these results with the laser-annealed sample first. The small ordered parts of this surface clearly do not resemble the $(7 \times 7)$ structure very closely at all. There is in fact a much closer correspondence with the equilibrium germanium surface on which we have recently reported. ${ }^{12}$ This surface is observed by LEED to take a $c-(2 \times 8)$ reconstruction but tunneling images also showed isolated fields of $(2 \times 2)$ and $c-(4 \times 2)$ that had previously gone undetected. In that work we pointed out that arrays of coherently arranged $c-(4 \times 2)$ and $(2 \times 2)$ cells can produce the $c-(2 \times 8)$ reconstruction. Problems that many people have had in producing high-quality $c-(2 \times 8)$ patterns on germanium may be caused by the difficulty the surface experiences in generating the long-range coherence from the smaller-area, higher-symmetry subreconstructions. We therefore propose that the laser-annealed silicon surface corresponds to that case with the rapid quench making it virtually impossible for the analogous long-range order to be achieved. We further suggest that this correspondence exists for the high-temperature equilibrium surface phase $\left(T>977^{\circ} \mathrm{C}\right)^{13}$ and suggest that careful high-temperature LEED studies should find evidence for it. On the other hand we may also anticipate that germanium may have a low-temperature $(7 \times 7)$ structure that has previously escaped detection.
The simplest microscopic explanation for both the $c-(4 \times 2)$ and the $(2 \times 2)$ structures can be constructed out of non-close-packed adatom models where only one type of threefold-symmetric surface site is populated. We prefer the "closed" site, i.e., that site having a bulk atom just below it at the bottom of the top double layer. The reason for this choice is the same as that discussed below for the $(\sqrt{3} \times \sqrt{3})$ case.

The partially thermally annealed surface data tells a completely different story. Here it should be clear that all deviations from $(7 \times 7)$ structures are still closely related to it. We believe that it is no accident that the currently popular dimer-adatom-stacking fault model proposed by Takayanagi et al. ${ }^{14}$ can be extended from the $(7 \times 7)$ to $(5 \times 5)$ and $(9 \times 9)$ structures in a straightforward way. In the $(7 \times 7)$ case the outermost complete double layer in the unit mesh consists of two triangular subunits that are respectively faulted and unfaulted relative to the substrate. These two types of regions are "zipped" together by a $60^{\circ}$ partial dislocation which contains the dimer structures mentioned in the model's name. Finally each triangular subunit is terminated with an outer layer of six adatoms over the so-called closed triangular sites. Deep holes at the corners of the rhombic unit mesh follow naturally from the above.

It is possible to extend the model to cover all $(2 n-1) \times(2 n-1)$ cases by shrinking or expanding the unit cell along the two primitive translation directions. The structures for $(5 \times 5),(7 \times 7)$, and $(9 \times 9)$ are illustrated in Fig. 4. Chadi ${ }^{15}$ has used the $(5 \times 5)$ as a computational aid in theoretical studies on silicon surfaces, and germanium-silicon alloys have recently yielded tunneling images that correspond to this structure. $^{16}$ The $(9 \times 9)$ is the first member of this class of

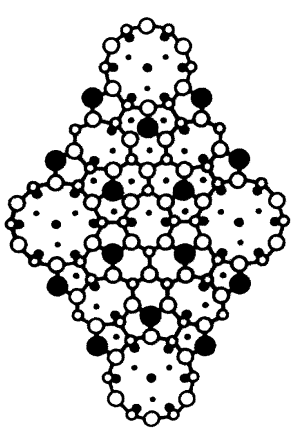

$5 \times 5$

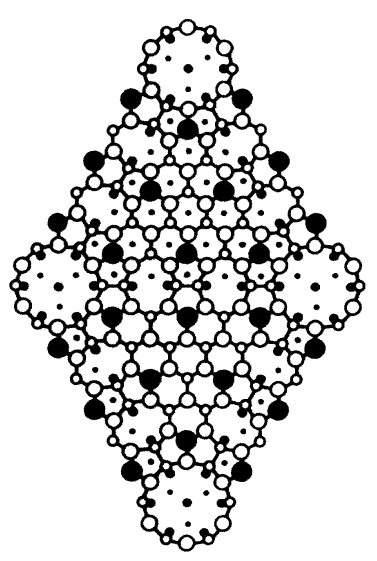

$7 \times 7$

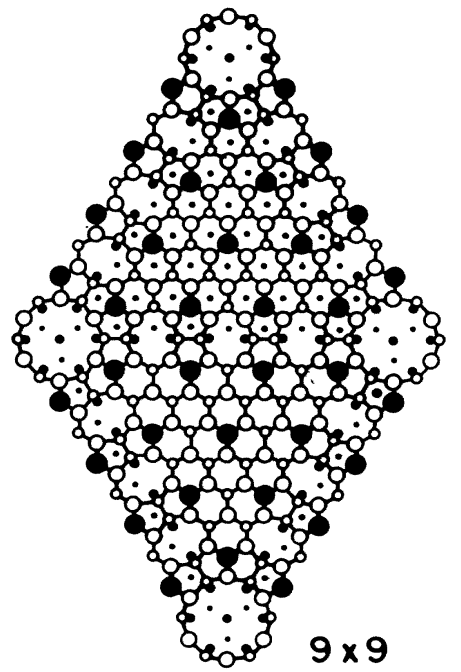

FIG. 4. Top view of $(5 \times 5),(7 \times 7)$, and $(9 \times 9)$ structures according to dimer-adatom-stacking fault model. Shaded large circles are adatoms. Open circles are atoms in the partially faulted double layers directly below. Filled circles are the bulk unreconstructed double layer of the bulk. 
structures that contains adatoms that are not adjacent to the sublayer partial dislocation which bounds each triangular half-cell. Electronic structure associated with this surface atom may be significantly different from the rest of the surface as a result and we are currently attempting to detect this effect. At this time we can only indicate that differential conductivity measurements show an asymmetry across the $(9 \times 9)$ unit-mesh short diagonal that confirms the presence of a stacking fault in a way we have already reported on for the $(7 \times 7) .^{9}$

It is also possible to comment in some detail on the $(\sqrt{3} \times \sqrt{3})$ region shown in Fig. 2 . Northrop ${ }^{17}$ has recently performed total-energy calculations for an adatom model of this structure in an attempt to understand why closed triangular sites seem to be preferred by silicon adatoms on the equilibrium $\mathrm{Si}$ (111) surface. He finds that significant relaxation of the atom directly below makes this a low-energy site. We therefore assign this nonequilibrium $(\sqrt{3} \times \sqrt{3})$ structure to a close-packed adatom surface populating all closed triangular double-layer sites.

It is difficult to end this discussion of new surface structure on silicon surfaces without pointing out that the high spatial resolution and specificity of the tunneling microscope has made it possible to see and study individual unit cells under conditions where long-range order does not exist. We are essentially doing crystallography on single unit cells. For those of us with experience in diffraction physics this is quite a transition from the study of systems which of necessity must have long-range order to be observed at all. The long-range properties of these systems tend to make us think of matter in terms of global properties and make conclusions, such as those reached here, from small regions seem somewhat bold. Nevertheless it may well be local rather than global properties that often play the major role in determining the properties of metastable or nonequilibrium systems such as those we have prepared and studied here. The explanation for the presence of the structures we have observed will probably lie in the defects, strain fields, and local energy barriers to structural transformation.

In conclusion the silicon (111) surface continues to be a fascinating interface to study. The five new reconstructions we have reported on here attest to the remarkable richness of structures that can compete and be accommodated.

We would like to express particular appreciation to D. H. Auston, V. Narayanamurti, and C. K. N. Patel for support of this work.

1D. M. Zehner, C. W. White, and G. W. Ownby, Appl. Phys. Lett. 36, 56 (1980), and Surf. Sci. 92, L67 (1980).

2P. L. Cowan and J. A. Golovchenko, J. Vac. Sci. Technol. 17, 1197 (1980).

${ }^{3}$ R. M. Tromp, E. J. van Loenen, M. Iwami, and F. Saris, Solid State Commun. 44, 971 (1982).

${ }^{4}$ D. M. Zehner, C. W. White, P. Heimann, B. Reihl, F. J. Himpsel, and D. E. Eastman, Phys. Rev. B 24, 4875 (1981).

${ }^{5}$ D. M. Zehner, J. R. Noonan, H. L. Davis, and C. W. White, J. Vac. Sci. Technol. 18, 852 (1981); F. Jona, P. M. Marcus, H. L. Davis, and J. R. Noonan, Phys. Rev. B 33, 4005 (1986).

${ }^{6}$ Y. J. Chabal, G. S. Higashi, and S. B. Christman, Phys. Rev. B 28, 4472 (1983).

${ }^{7}$ Y. J. Chabal, J. E. Rowe, and D. A. Zwemer, Phys. Rev. Lett. 46, 600 (1981); W. S. Yang and F. Jona, Phys. Rev. B 28, 1178 (1983).

${ }^{8}$ G. Binnig, H. Rohrer, V. Gerber, and E. Weibel, Phys. Rev. Lett. 46, 600 (1981). For a recent review, see J. A. Golovchenko, Science 232, 48 (1986).

${ }^{9}$ R. S. Becker, J. A. Golovchenko, E. G. McRae, and B. S. Swartzentruber, Phys. Rev. Lett. 55, 2028 (1985); R. S. Becker, J. A. Golovchenko, D. R. Hamann, and B. S. Swartzentruber, Phys. Rev. Lett. 55, 2032 (1985).

${ }^{10}$ D. H. Auston, J. A. Golovchenko, A. L. Simons, C. M. Surko, and T. N. C. Venkatessan, Appl. Phys. Lett. 34, 777 (1979).

${ }^{11}$ C. M. Surko, A. L. Simons, D. H. Austin, J. A. Golovchenko, R. E. Slusher, and T. N. C. Venkatessan, Appl. Phys. Lett. 34, 635 (1979).

${ }^{12}$ R. S. Becker, J. A. Golovchenko, and B. S. Swartzentruber, Phys. Rev. Lett. 54, 2678 (1985).

${ }^{13}$ H. D. Hagstrum and G. E. Becker, Phys. Rev. B 8, 1580 (1973); P. A. Bennet and M. W. Webb, Surf. Sci. 104, 74 (1981)

${ }^{14} \mathrm{~K}$. Takayanagi, Y. Tanishiro, M. Takahashi, and S. Takahashi, J. Vac. Sci. Technol. A 3, 1502 (1985).

${ }^{15}$ D. J. Chadi, Phys. Rev. B 30, 4470 (1984).

${ }^{16}$ R. S. Becker, J. A. Golovchenko, and B. S. Swartzentruber, Phys. Rev. B 32, 8455 (1985).

17J. Northrup, Bull. Am. Phys. Soc. 31, 584 (1986). 


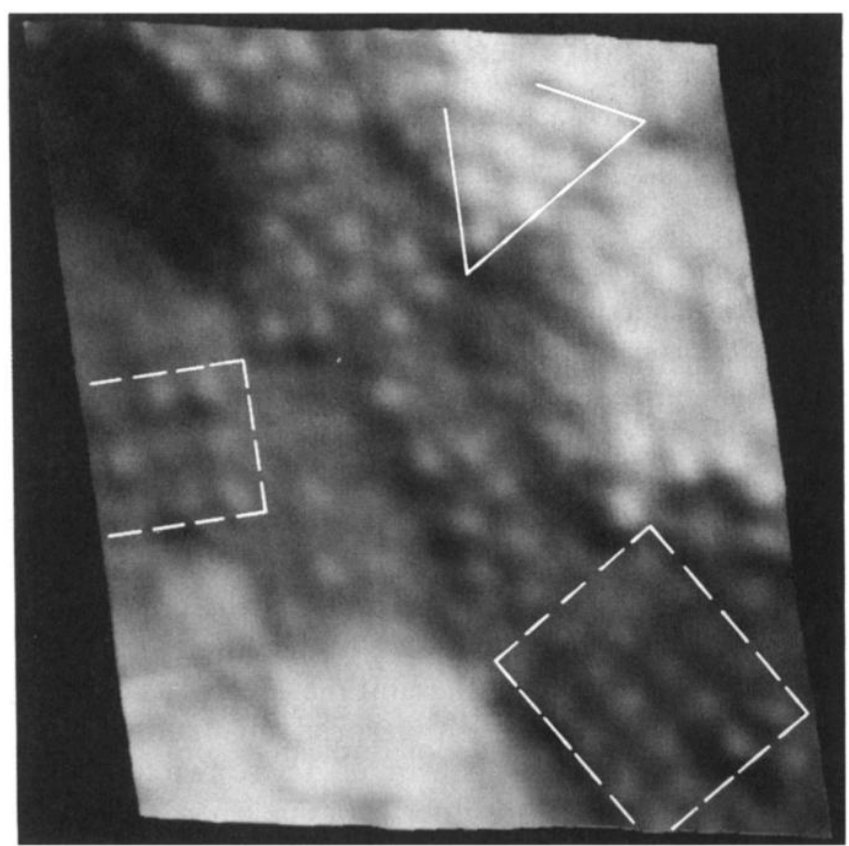

FIG. 1. Tunneling image of a laser-annealed Si (111) surface. Dashed lines enclose regions of $c-(4 \times 2)$ reconstruction and solid lines delineate $(2 \times 2)$ regions. Shading is by height with light areas high and dark areas low. The two light-gray regions in the lower left and upper right are double-layer atomic steps $(3.1 \AA)$ up from the gray regions containing the $c-(4 \times 2)$. The area shown is $\sim 95 \times 105 \AA^{2}$. The tip bias was $-0.5 \mathrm{~V}$ and tunnel current $1.0 \mathrm{nA}$. Image acquisition time is $2 \mathrm{~min}$. 


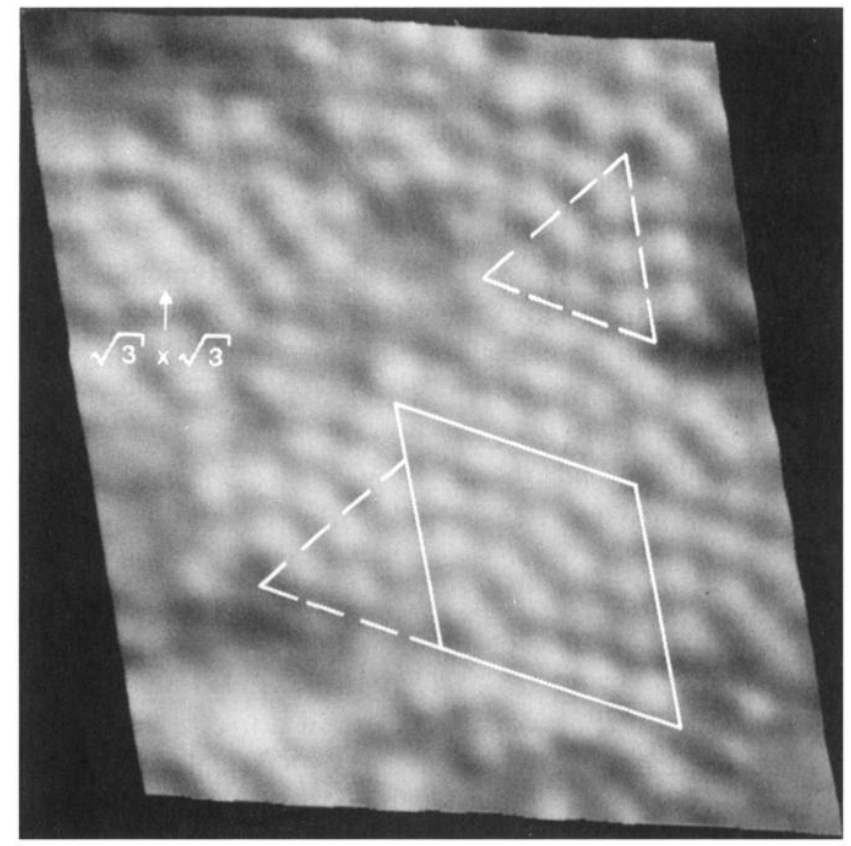

FIG. 2. Tunneling image of a laser-annealed Si (111) surface subsequently thermally annealed at $600{ }^{\circ} \mathrm{C}$. The dashed lines indicate partial $(7 \times 7)$ cells. The solid line surrounds a $(9 \times 9)$ cell. A $(\sqrt{3} \times \sqrt{3})-R 30$ region is indicated in the upper left. The area depicted is $\sim 95 \times 105 \AA^{2}$ and the gray areas are assigned as in Fig. 1. 


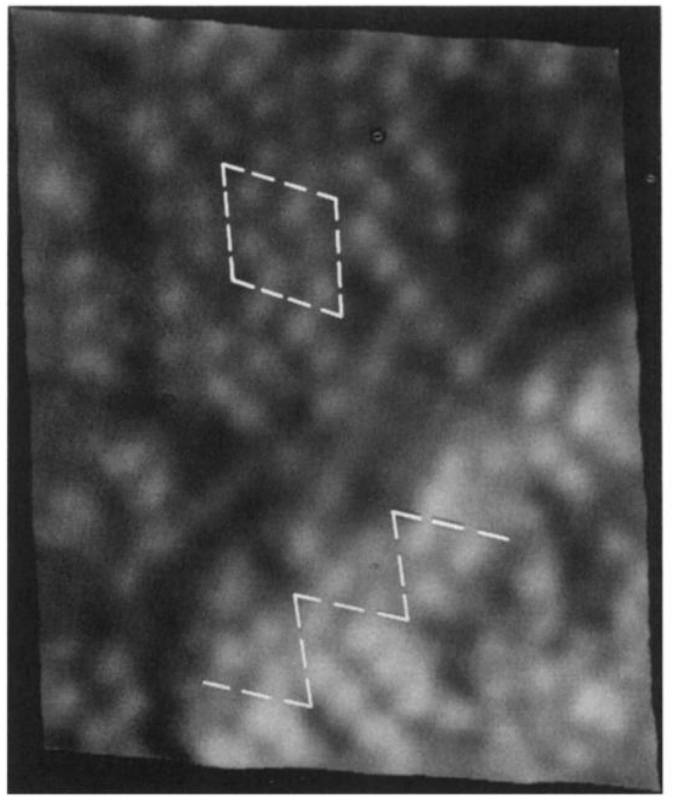

FIG. 3. Tunneling image of a laser-annealed $\mathrm{Si}$ (111) surface subsequently thermally annealed at $600^{\circ} \mathrm{C}$. The area depicted is $\sim 95 \times 105 \AA^{2}$. The dashed lines depict $(5 \times 5)$ cells and half-cells which are prominent along the step edge at the lower right. Gray levels assigned as in Fig. 1. 